\title{
Mid-infrared spectroscopy of UV irradiated hydrogenated amorphous carbon materials
}

\author{
K. A. K. Gadallah ${ }^{1,2}$, H. Mutschke ${ }^{1}$, and C. Jäger ${ }^{3}$ \\ 1 Astrophysical Institute and University Observatory, Friedrich Schiller University, Schillergässchen 2/3, 07743 Jena, Germany \\ e-mail: Kamel.Gadallah71@gmail.com \\ 2 Astronomy Dpt., Faculty of Science, Al-Azhar University, Nasr City, PO Box 11884 Cairo, Egypt \\ ${ }^{3}$ Institute of Solid State Physics, Friedrich Schiller University of Jena, Helmholtzweg 3, 07743 Jena, Germany
}

Received 20 March 2012 / Accepted 22 June 2012

\section{ABSTRACT}

\begin{abstract}
Context. Mid-infrared (MIR) bands are characteristic for the short-range and medium-range structure of hydrogenated amorphous carbon (HAC) materials that exist in the interstellar medium (ISM) and are sensitive to processing in the harsh interstellar radiation field.

Aims. We study the variability of the MIR features from the spectrum of non-processed to that of UV-processed HAC materials and compare them to spectra of interstellar carbonaceous materials.

Methods. Nano-sized HAC materials produced by laser ablation were irradiated by vacuum-UV photons with doses comparable to those relevant for interstellar processing. They were subsequently analyzed by IR spectroscopy.

Results. In the MIR range, the spectra of HAC materials show many absorption bands such as the $\mathrm{sp}^{3}$ aliphatic $\equiv \mathrm{C}-\mathrm{H}$ stretching vibration at $3.03 \mu \mathrm{m}$, the $\mathrm{sp}^{3}$ aliphatic $-\mathrm{C}-\mathrm{H}$ stretching vibration at $3.4 \mu \mathrm{m}$ and also the $\mathrm{sp}^{3}$ aliphatic $\mathrm{C}-\mathrm{H}$ bending vibration at both $6.85 \mu \mathrm{m}$ and $7.25 \mu \mathrm{m}$. All these are recognizable bands of HAC materials. Other absorption bands such as the sp ${ }^{2}$ aromatic $=\mathrm{C}-\mathrm{H}$ stretching vibration at about $3.3 \mu \mathrm{m}$ and the $\mathrm{sp}^{2}-\mathrm{C}=\mathrm{C}$ stretching vibration close to $6.25 \mu \mathrm{m}$ are observed. The HAC materials also possess bands which represent the aromatic out-of-plane bending at $11.65,12.46$ and $12.9 \mu \mathrm{m}$ in addition to the aromatic $-\mathrm{C}-\mathrm{C}-\mathrm{C}$ in-plane bending at $15.87 \mu \mathrm{m}$. With UV irradiation, the mass absorption coefficient of the $3.03 \mu \mathrm{m}$ band completely disappears and that of the aliphatic $\mathrm{C}-\mathrm{H}$ bands $(3.4,6.85$ and $7.25 \mu \mathrm{m})$ decreases. This reduction shows that the UV radiation destroys most of the aliphatic $\mathrm{C}-\mathrm{H}$ bonds inside the HAC structure. On the other hand, the strength of the aromatic $6.2 \mu \mathrm{m}$ band increases, which is evidence of the partial graphitization within UV-irradiated HAC materials. Because UV irradiation is not uniform, this band agrees well with the C-class PAH toward HD 100764. The C-H out-of-plane vibration bands are strongly affected by UV irradiation. Bands at 11.35 , 12.14 and $12.64 \mu \mathrm{m}$ (solo, duo and trio, respectively), which are found for PAHs of many interstellar spectra were observed partially and were compared to those of non-processed materials, in particular, those at $11.35 \mu \mathrm{m}$, which represent the aromatic structures.

Conclusions. UV irradiation has a variable effect on both aliphatic and aromatic bands in the MIR region. The aliphatic C-H structure decreases while the aromatic $\mathrm{C}=\mathrm{C}$ structure, which might lead to the graphitic colonies for PAHs, increases. UV irradiation has revealed solo, duo and trio bands that are relatively consistent with those of the A- and B-class PAHs.
\end{abstract}

Key words. ultraviolet: ISM - methods: laboratory - dust, extinction - astrochemistry - ISM: clouds - infrared: ISM

\section{Introduction}

The effect of highly energetic UV irradiation on the bonding inside the structure of the hydrocarbons can be analyzed by mid-infrared (MIR) spectroscopy. An extremely important component of the carbonaceous matter in the diffuse interstellar medium (ISM) could be the hydrogenated amorphous carbon (HAC) material. In the Milky Way, these materials have been observed at the $3.4 \mu \mathrm{m}$ stretching band along many lines of sight in the local diffuse ISM (Pendleton et al. 1994; Whittet et al. 1997; Pendleton \& Allamandola 2002) and along lines of sight of Sgr A*, GCS 3 and GCS 4 toward the galactic center (Chiar et al. 2000, 2002). They are also observed at $6.8 \mu \mathrm{m}$ and $7.25 \mu \mathrm{m}$ (C-H deformation bands) in Spitzer IRS extragalactic spectra in the diffuse ISM of many galaxies (Dartois \& Muñoz-Caro 2007). These IR vibrational bands are very sensitive to the bonding types of $\mathrm{C}$ atoms $\left(\mathrm{sp}^{1}, \mathrm{sp}^{2}\right.$ and $\mathrm{sp}^{3}$ hybridization) in a carbonaceous material. Therefore, MIR spectra of HACs are characterized by their aromatic and aliphatic substructures. Astronomical observations in the MIR range have shown that many specific features refer to the presence of aromatic and aliphatic compounds in ISM. These features provide information about the composition and evolution of interstellar carbonaceous dust grains that are exposed to the interstellar irradiation field, including the exposure to UV photons, heating, and ion irradiation.

The group of unidentified infrared bands (UIBs) at 3.3, 6.2, 7.7, 8.6, and $11.3 \mu \mathrm{m}$ has been commonly observed in a wide variety of astronomical environments such as the planetary nebulae (NGC 7027), the photodissociation region at the Orion Bar, and the nuclear region of NGC 4536 (Peeters 2011). These bands are attributed to various vibrational modes of aromatic hydrocarbon bonds. There are various carbonaceous materials that can show these bands, for instance gas-phase molecules such as polycyclic aromatic hydrocarbons (PAHs, Puget \& Leger 1989; Allamandola et al. 1989), quenched carbonaceous composite (Sakata et al. 1987), and carbonaceous solids such as coal (Papoular et al. 1989). The high temperatures required for the emission of these bands, however, indicates that their carriers are more probably large molecules (PAHs) and not solid particles, because they need to be able to be stochastically heated by single photons (Draine \& Li 2001). 
Observations of several extreme carbon stars and C-rich protoplanetary nebulae (proto-PN) performed by Kwok et al. (1999) have shown that the strengths of the 3.4, 6.9, 12.1, 12.4, and $13.3 \mu \mathrm{m}$ features decrease relative to those of the $3.3,6.2$, and $11.3 \mu \mathrm{m}$ features when stars evolve from proto-PN to PN. This means that there is a transformation from aliphatic to aromatic structures resulting in larger aromatic units. In the range of $10-15 \mu \mathrm{m}$, the spectra have revealed emission features at 11.2 , 12.7, 13.5, and $14.2 \mu \mathrm{m}$ (Hony et al. 2001; Sloan et al. 2005) in different astrophysical environments. They are caused by $\mathrm{CH}$ out-of-plane bending modes of PAHs. Laboratory studies of thin HAC films assembled from carbon nanoparticles conducted by Hu \& Duley (2007) have shown that the spectra in the range from $11 \mu \mathrm{m}$ to $15 \mu \mathrm{m}$ show features similar to the interstellar emission bands of PAHs at 11.3, 12, 12.8, 13.5, and $14.2 \mu \mathrm{m}$. They suggested that these bands might be attributed to compact ring structures such as coronene $\left(\mathrm{C}_{24} \mathrm{H}_{12}\right)$, which has peripheral substitutions with additional aromatic rings or $\mathrm{sp}, \mathrm{sp}^{2}$-bonded carbon chains.

Sloan et al. (2007) have shown that the IR emission bands in the range $5-14 \mu \mathrm{m}$ are caused by aromatic and aliphatic structural units. These features vary in strength and position from one object to the next as described by the classification scheme introduced by Peeters et al. (2002). In this scheme, the spectra of PAHs have been classified into A-, B- and C-classes depending on the peak position and width of the $6.2 \mu \mathrm{m}$ band related to the shift of the position of 7.7 and $8.6 \mu \mathrm{m}$ bands. The peak position of these classes falls in ranges of 6.19-6.23, 6.235-6.28 and up to $6.35 \mu \mathrm{m}$, respectively. The width of the B-class is wider than that of the other classes. These authors found that spectra of the known C-class PAHs are excited by relatively cool stars and suggested that carbonaceous materials are formed as large HAC conglomerates. Once these conglomerates are ejected into a harsh radiation field, their aliphatic bonds are subsequently broken and the PAH features appear over time. This study shows that hydrocarbons toward objects with $\mathrm{C}$-class $\mathrm{PAH}$ are relatively protected while those toward objects with A and B-class PAHs are more processed by more energetic photons.

Under the conditions of diffuse interstellar clouds $\left(A_{V}=\right.$ 0.6 ), the average interstellar UV radiation flux is about $3 \times$ $10^{8} \mathrm{eV} \mathrm{cm}^{-2} \mathrm{~s}^{-1}$. Hence the total average interstellar dose of UV photons deposited on HAC grains is $3 \times 10^{23} \mathrm{eV} \mathrm{cm}^{-2}$ (Jenniskens 1993) during the lifetime of the diffuse cloud $(3 \times$ $10^{7} \mathrm{yr}$, see Sorrell 1990, for more details).

In the recent paper by Gadallah et al. (2011) it has been shown that HAC materials can also produce one of the strongest IR bands as an analog to the $3.4 \mu \mathrm{m}$ band in the diffuse ISM. In addition, UV irradiation of HAC can form a new band in the UV similar to the interstellar UV bump at $217.5 \mathrm{~nm}$. In this paper, which can be considered an extension of our previous study (Gadallah et al. 2011) on the effect of UV processing on HAC materials in the UV and visible range, we discuss the spectra of non-processed and UV-processed HAC materials in the MIR range including the spectral features at 3.4, 6.2, 6.8 , and $7.25 \mu \mathrm{m}$ in addition to others in the fingerprint region at $11.6,12.4,13.1$ and $13.9 \mu \mathrm{m}$ and their development after the UV processing.

\section{Experiments and Fourier transformation infrared spectroscopy}

From our previous work (Gadallah et al. 2011), which described the role of the UV irradiation of HACs in the UV-VIS spectral

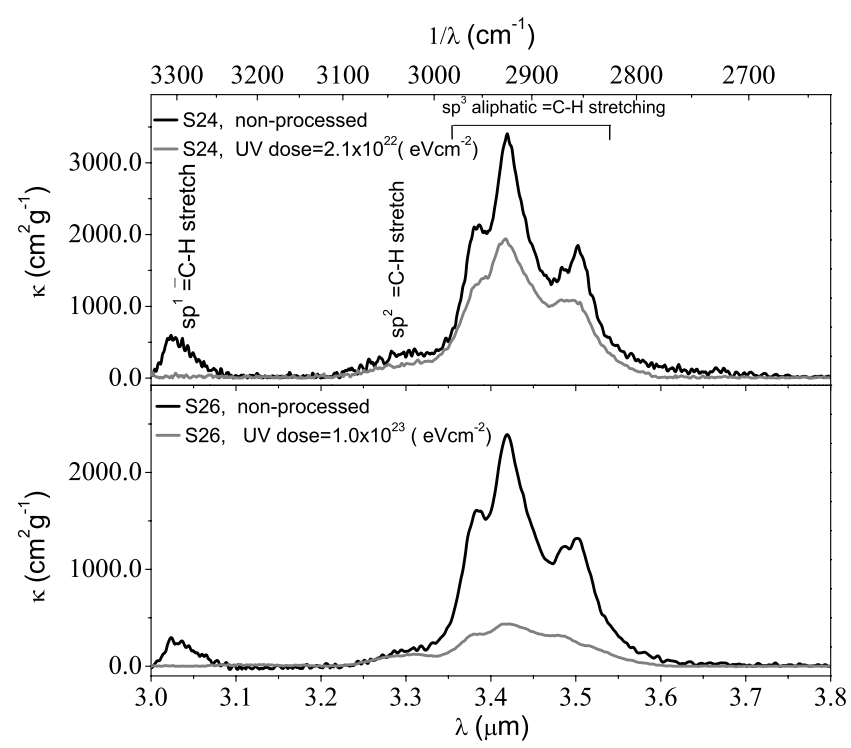

Fig. 1. Experimental spectra of the C-H stretching band of samples (S24 and S26) before and after UV irradiation.

region, two samples (S24 and S26 in Table 1 of that paper) were taken into account to explain the same role in the MIR spectral region. These samples were produced by the laser ablation method of a rotating graphite target in a quenching gas atmosphere $\left(\mathrm{He} / \mathrm{H}_{2}\right)$ at a pressure of $4.5 \mathrm{mbar}$. The $\mathrm{He} / \mathrm{H}_{2}$ ratio is 4.5 and 2.5 for S24 and S26, respectively. In this method, a $\mathrm{Nd}$ :YAG laser with a wavelength of $532 \mathrm{~nm}$ was used. Its power is $2.8 \times 10^{10} \mathrm{Wcm}^{-2}$ to produce S24 and $3.1 \times 10^{10} \mathrm{Wcm}^{-2}$ to produce S26. These higher values of the laser power can vaporize very small quantities of graphite in a form of intense plasma in which the temperature is higher than $4000 \mathrm{~K}$ (Iida \& Yeung 1994). Collisions between the evaporated carbon components with the quenching gas in a supersonic expansion of the hot plasma cause the condensation of carbon particles. S24 and S26 were deposited in a separated chamber (substrate chamber, please see Fig. 1 in Jäger et al. 2008, for details) at a pressure of $(5 \pm 3) \times 10^{-6}$ Torr on $\mathrm{CaF}_{2}$ and $\mathrm{KBr}$ substrates, respectively. Quantitatively, the atomic and electronic structures of these two samples are different. The $\mathrm{H} / \mathrm{C}$ ratio of $\mathrm{S} 24$ is 0.82 while that of S26 is 0.75 . The hybridization $\left(\mathrm{sp}^{3} / \mathrm{sp}^{2}\right)$ is 0.8 and 0.65 , respectively. The variation in each ratio reveals that $\mathrm{S} 24$ has a much more aliphatic structure than S26. Gadallah et al. (2011) found that the ratios of the $\mathrm{H} / \mathrm{C}$ and $\mathrm{sp}^{3} / \mathrm{sp}^{2}$ were reduced with UV irradiation $(0.70$ and 0.56 , respectively, for $\mathrm{S} 24$, and 0.37 and 0.14 , respectively, for S26). These samples were processed by $\mathrm{UV}$ radiation up to $2.1 \times 10^{22}$ and $1.0 \times 10^{23} \mathrm{eV} \mathrm{cm}^{-2}$ respectively, using a deuterium lamp (L2D2 lamp, type L7292, from HAMAMATSU) at the wavelength $160 \mathrm{~nm}$. These doses correspond to $7 \%$ and $33 \%$ of those of the diffuse ISM, respectively.

Because the Fourier transformation IR spectroscopy (FTIR) is based on the vibrations of the atoms of a molecule, it can be used to study the chemical bonding of hydrogen and its concentration in HAC material. The possible vibrational modes of HAC materials (or carbon soot) have been tabulated by e.g., Dischler et al. (1983) and Jäger et al. (2008). The IR transmission measurements were taken in-situ (ex situ for S24 after UV processing) by using the FTIR spectrometer Bruker $113 \mathrm{v}$. They were corrected directly during the measurements by using a blank substrate as reference for the transmission spectrum for each sample. A baseline correction of all spectra shown in the 
Table 1. Gaussian fitting of the spectra of HAC materials (S26) before and after UV irradiation.

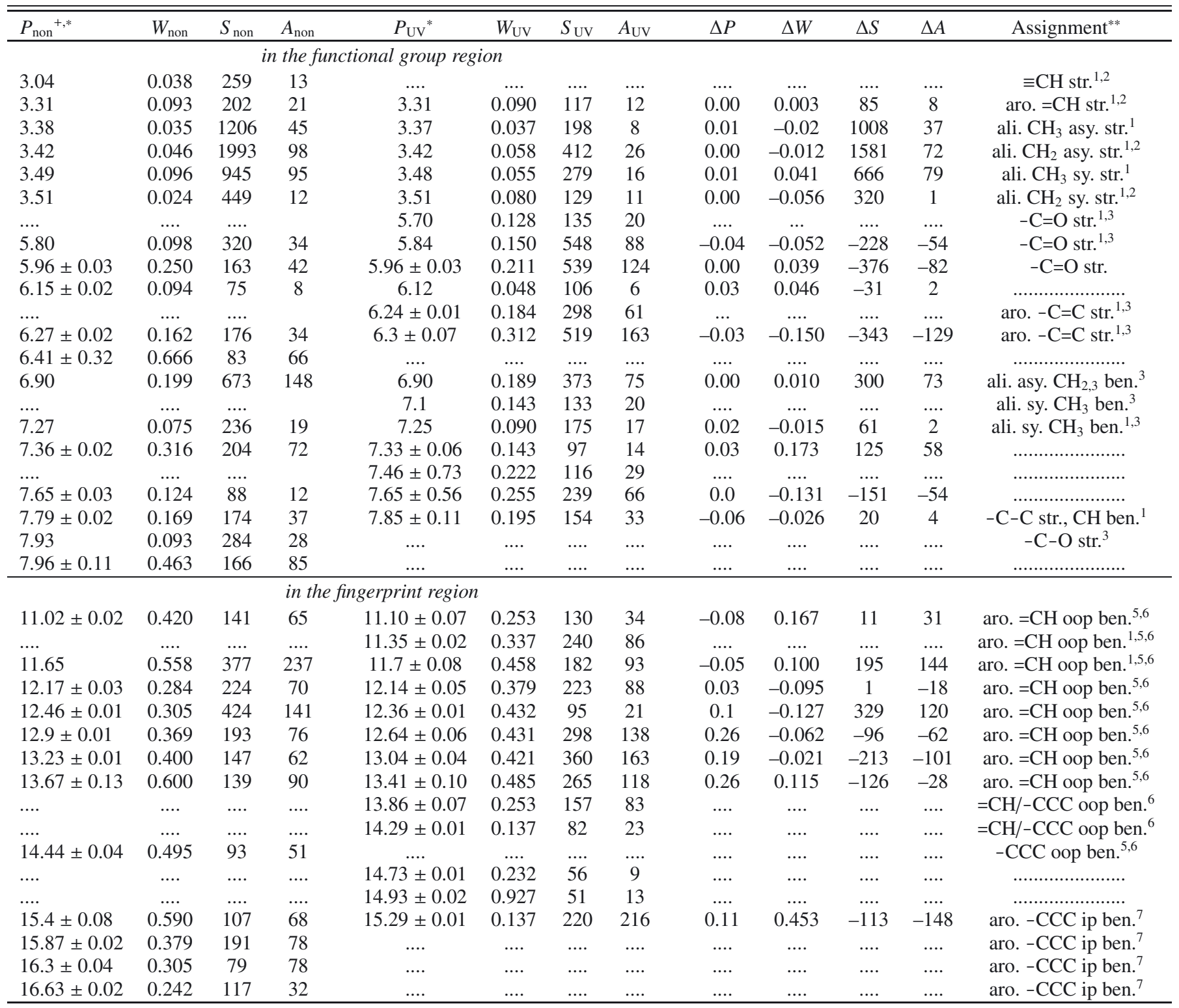

Notes. ${ }^{(+)}$In the first row, we list the peak parameters (the position $P(\mu \mathrm{m})$, width $W(\mu \mathrm{m})$, strength $S\left(\mathrm{~cm}^{2} \mathrm{~g}^{-1}\right)$ and integrated area $\left.A\right)$ and their changes $\left(\Delta P, \Delta W, \Delta S\right.$ and $\Delta A$, respectively). The subscripts non and $U V$ refere to the non- and UV-processed samples ${ }^{(*)}$ In the first and fifth columns, we list the errors estimates $(\geq 0.01 \mu \mathrm{m})$ of the band position. ${ }^{(*)}$ In the last column, the abbreviations of aro., ali., str., ben., asy., sy., oop, and ip refer to aromatic, aliphatic, bending, asymetric, symetric, out-of-plane, and in-plane, respectively.

References. (1) Jäger et al. (2008); (2) Dischler et al. (1983); (3) Grishko \& Duley (2002); (4) Mckenzie et al. (1983); (5) Hudgins \& Allamandola (1999); ${ }^{(6)}$ Hony et al. (2001); (7) Van Kerckhoven et al. (2000).

next figures was performed in order to subtract the continuum absorption.

\section{Results}

The IR absorption spectra provide much information about the hydrogen content and the $\mathrm{C}-\mathrm{H}$ bonding. The stretching modes of $\mathrm{C}-\mathrm{H}_{n}(n=1,2,3)$ groups originate from $\mathrm{sp}^{1}, \mathrm{sp}^{2}$, and $\mathrm{sp}^{3}$ hybridized carbon. Their strength depends on the preparation conditions. These bands are observed in the region from 3.0 to $3.57 \mu \mathrm{m}$ in the spectrum of HAC materials. The $\mathrm{sp}^{3} / \mathrm{sp}^{2}$ ratio can be calculated from the hydrogen content, which is derived from the integrated area under these stretching bands (Angus \& Jansen 1988; Gadallah et al. 2011). In addition, the bending (C-H deformation) modes are also observed at 6.85 and $7.25 \mu \mathrm{m}$ in the spectrum of HAC materials.

Here, the MIR range was divided into a region from $3 \mu \mathrm{m}$ to $8 \mu \mathrm{m}$ (the functional group region) and into another region from $10 \mu \mathrm{m}$ to $17 \mu \mathrm{m}$ (the fingerprint region). Figures 1 and 2 show the spectra for samples (S24 and S26) in the functional group region of non-processed and UV-processed materials, while Fig. 3 shows those in the fingerprint region for the sample S26. The Gaussian fitting of the spectrum of S26 in these figures is shown in Figs. 4-6. In the Gaussian fitting, we selected a certain number of the most noticeable peaks that give 


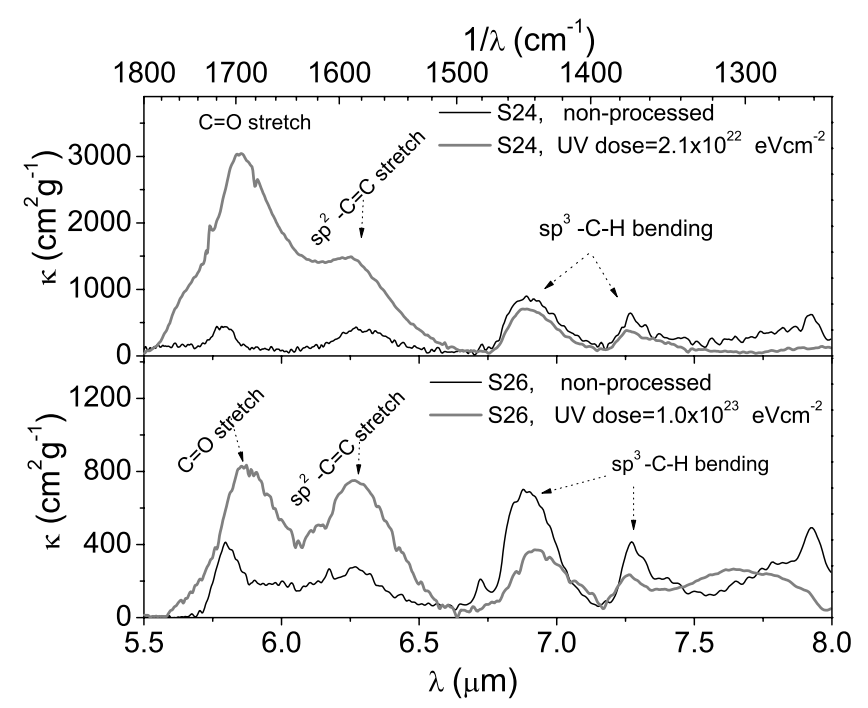

Fig. 2. Experimental spectra in the range from 5.5 to $8 \mu \mathrm{m}$ of samples (S24 and S26) before and after UV irradiation.

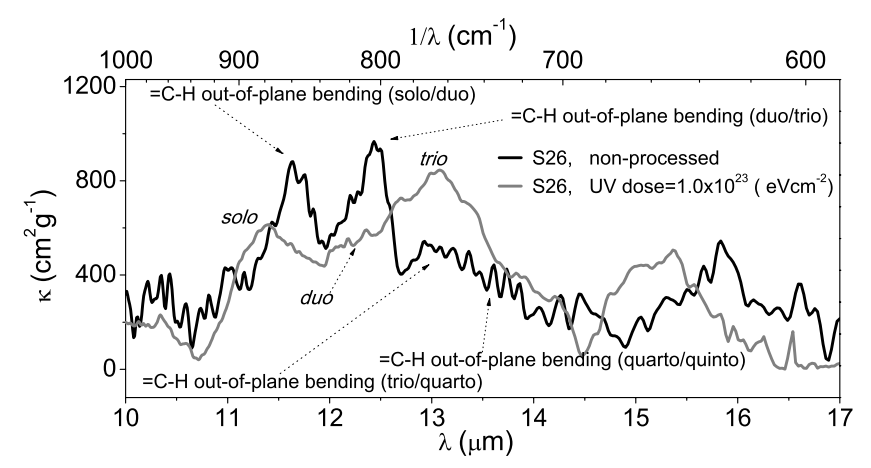

Fig. 3. Experimental spectra in the range from 10 to $17 \mu \mathrm{m}$ of sample S26 before and after UV irradiation.

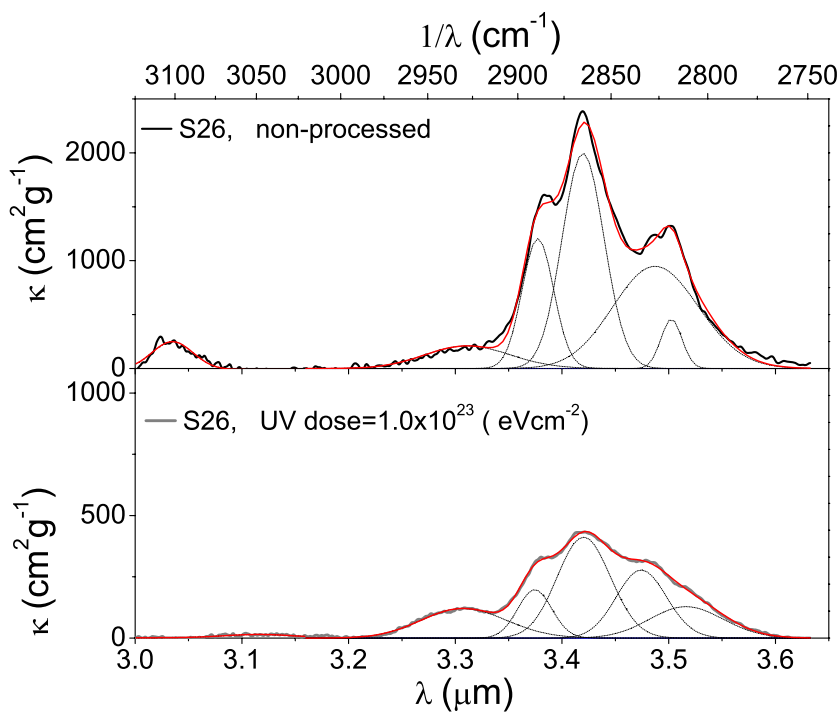

Fig. 4. Analysis by Gaussian fitting of the non-processed and UV-processed spectra of S26, in the $\mathrm{C}-\mathrm{H}$ stretching region.

the best fitting of the accumulative curve. Indeed, as this number increases, the line shape fits well, therefore our fitting may not give a unique solution for the fitting analysis. The fitting parameters are listed in Table $1 . P_{\text {non }}, W_{\text {non }}, S_{\text {non }}$ and $A_{\text {non }}$ refer to the

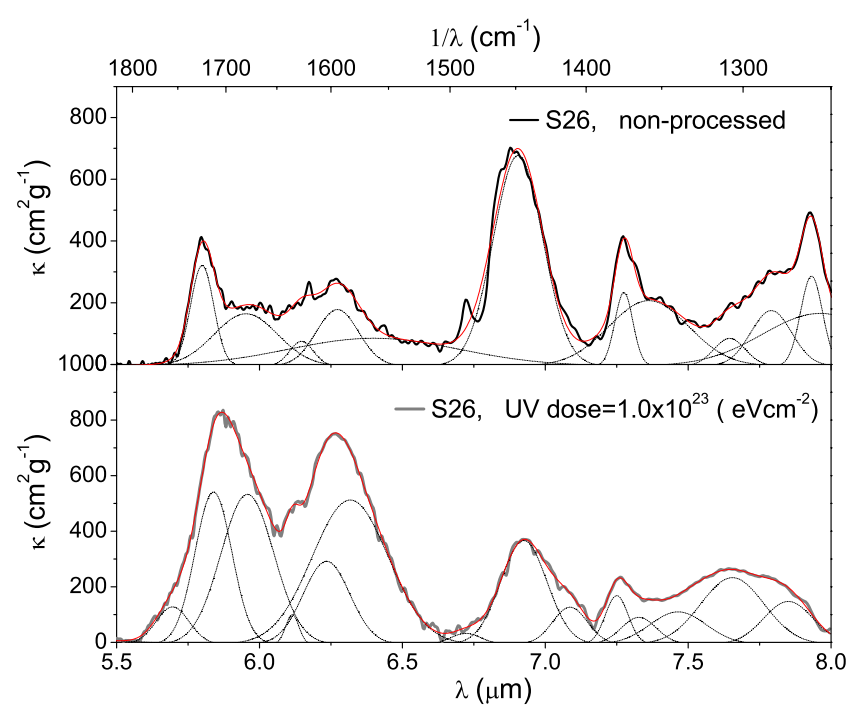

Fig. 5. Analysis by Gaussian fitting of the spectra in the range from 5.5 to $8 \mu \mathrm{m}$ of $\mathrm{S} 26$ before and after UV irradiation.

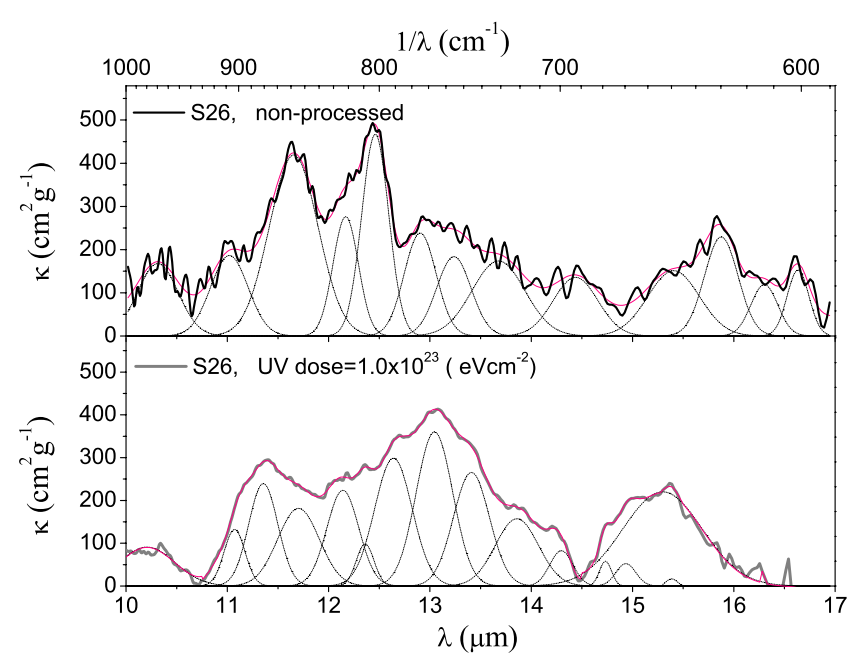

Fig. 6. Analysis by the Gaussian fitting of the spectra in the range from 10 to $17 \mu \mathrm{m}$ of S26 before and after UV irradiation.

position, width, strength, and integrated area of bands of nonprocessed samples, while $P_{\mathrm{UV}}, W_{\mathrm{UV}}, S_{\mathrm{UV}}$ and $A_{\mathrm{UV}}$ refer to those of the bands of UV-processed samples. The position and width are termed in the wavelength, while the strength is the maximum value of the mass absorption coefficient $\kappa$. The integrated intensity of a band is represented by the integrated area of that band. The changes of the position, width, strength, and integrated band intensity are represented by $\Delta P\left(P_{\text {non }}-P_{\mathrm{UV}}\right), \Delta W\left(W_{\text {non }}-W_{\mathrm{UV}}\right)$, $\Delta S\left(S_{\text {non }}-S_{\mathrm{UV}}\right)$ and $\Delta A\left(A_{\text {non }}-A_{\mathrm{UV}}\right)$, respectively. The negative values of these variations imply that the position, strength, width, and integrated intensity of the bands increase while positive values refer to a decrease of that parameters. We observe from the values of $\Delta S$ different trends of the strength after the UV irradiation. The strength of all aliphatic bands (stretching or bending) has one direction. It decreases with the UV irradiation. On the other hand, the strength of the aromatic bands has different trends. For the aromatic $\mathrm{C}=\mathrm{C}$ stretching band group, it increases with UV irradiation. Although for the aromatic $=\mathrm{C}-\mathrm{H}$ (stretching or bending) group the bands decrease for all bands lower than or equal $12.36 \mu \mathrm{m}$, it increases for bands higher than $12.36 \mu \mathrm{m}$. For $-\mathrm{C}=\mathrm{O}$ stretching group bands, it becomes stronger. The trend 
of the band width variation, whatever its increases or decreases, does not factually reveal the effect of the UV radiation on the spectra of irradiated HAC materials. Some aliphatic bands such as 3.37 and $3.42 \mu \mathrm{m}$ are reduced very much while their width becomes broader, and sometimes this broadening may increase their integrated band intensities. For the $-\mathrm{C}=\mathrm{O}$ and aromatic $=\mathrm{C}-$ $\mathrm{H}$ group bands, there is no a standard trend of the width variation. The exception is the width of the aromatic $\mathrm{C}=\mathrm{C}$ stretching band, which becomes wider after the UV irradiation.

Some of bands listed in Table 1 are attributed to $\mathrm{sp}^{2}$ carboncarbon bonds and others are attributed to hydrogen atoms bonded to $\mathrm{sp}^{1}, \mathrm{sp}^{2}$, and $\mathrm{sp}^{3}$ carbon sites (e.g. Jäger et al. 2008; Dischler et al. 1983). The following list describes some of the most important vibrational modes in the spectrum of nonprocessed HAC materials (S26).

- The band at $3.04 \mu \mathrm{m}$ represents the stretching vibration of the $\mathrm{sp}^{1}$ aliphatic $\equiv \mathrm{C}-\mathrm{H}$ bonds such as in alkyne compounds.

- The band at $3.31 \mu \mathrm{m}$ is caused by the $\mathrm{sp}^{2}=\mathrm{C}-\mathrm{H}$ stretching vibrations. Its peak is relatively weak.

- The 3.38, 3.42, 3.49 and $3.5 \mu \mathrm{m}$ bands are the $\mathrm{sp}^{3}$ aliphatic $-\mathrm{C}-\mathrm{H}$ stretching vibration bands of $\mathrm{C}-\mathrm{H}$ groups (e.g. alkane $-\mathrm{C}-\mathrm{H}$ ) in aliphatic compounds (see Table 1). These modes are recognizable evidence of HAC materials.

- At about the position at $5.68 \mu \mathrm{m}$, there are the $-\mathrm{C}=\mathrm{O}$ bond stretching vibrations of all carbonyl compounds, to which belong the strong absorption at $5.8 \mu \mathrm{m}$ and other neighboring narrow bands of different carbonyl compounds. Mostly, these bands are caused by the incorporation of remaining oxygen (or oxygen-bearing molecules) during the laser ablation and condensation, although the materials were deposited in vacuum at $5 \pm 3 \times 10^{-6}$ Torr. In addition, combination bands of PAHs are expected to contribute in this range.

- The broad band at $6.2 \mu \mathrm{m}$ is resulting from the $\mathrm{sp}^{2}$ aromatic $\mathrm{C}=\mathrm{C}$ stretching vibration.

- The bands close to 6.85 and $7.25 \mu \mathrm{m}$ are also recognizable peaks of HAC materials representing the $\mathrm{sp}^{3}$ aliphatic $-\mathrm{C}-\mathrm{H}$ bending vibrations such as in alkanes.

- Bands located in the fingerprint region (from 10 to $17 \mu \mathrm{m}$ ) are the recognizable $=\mathrm{CH}$ out-of-plane bending vibrations of $\mathrm{sp}^{2}$ aromatic rings. Among them are the strong bands (solo/duo) close to $11.65 \mu \mathrm{m}$ for adjacent peripheral $1-2 \mathrm{H}$ atoms and the strong band (duo/trio) located near $12.46 \mu \mathrm{m}$ for adjacent peripheral 2-3 $\mathrm{H}$ atoms. A broad shoulder of trio/quarto/quinto bands were observed from 12.9 to $13.67 \mu \mathrm{m}$ for adjacent peripheral $3-5 \mathrm{H}$ atoms. There is also another strong band of the $\mathrm{sp}^{2}$ aromatic $-\mathrm{C}-\mathrm{C}-\mathrm{C}$ inplane bending which is located at about $15.87 \mu \mathrm{m}$.

Figure 1 shows that the UV irradiation has completely removed the stretching vibration of the $\mathrm{sp}^{1}$ aliphatic $\equiv \mathrm{C}-\mathrm{H}$ bonds at $3.03 \mu \mathrm{m}$. It turns out that this kind of bonding is easily broken up by the UV irradiation. Carbon-hydrogen single bonds $(-\mathrm{C}-\mathrm{H})$ can be dissociated by UV radiation, too, but long-time irradiation is needed. Analyses using the Gaussian fitting have shown that the strengths of the mass absorption coefficient have been changed after the UV irradiation as shown in Figs. 4-6. Some of the band positions are slightly shifted. Individual features in the spectra of non-processed and irradiated HAC materials will be discussed in detail in the following subsections.

\subsection{The 3-8 $\mu \mathrm{m}$ spectral range}

The $\mathrm{C}-\mathrm{H}$ stretching vibrations in the vicinity of the $3.4 \mu \mathrm{m}$ band include several modes of different functional groups containing
$\mathrm{C}-\mathrm{H}$ bonds. As we described above, UV irradiation removes many hydrogen atoms from the HAC materials. This is very clear from the reduction in strength of the aliphatic (symmetric and asymmetric) stretching vibrations of the functional groups $\mathrm{CH}_{2,3}$ at the whole $3.4 \mu \mathrm{m}$ band. The strength of the mass absorption coefficient decreases very much, as shown in Fig. 1, in particular in the spectrum of the processed S26 (with the highUV irradiation dose) compared to the spectrum of the processed S24 (with the low-UV irradiation dose). On the other hand, the strength of the aromatic $=\mathrm{C}-\mathrm{H}$ stretching vibrations at $3.31 \mu \mathrm{m}$ decreases with UV irradiation, too, but not by as much as that of its aliphatic neighbors. The fitting parameters in Table 1 show that the band positions at $3.38 \mu \mathrm{m}$ and $3.49 \mu \mathrm{m}$ are slightly moved toward shorter wavelengths after UV irradiation. For the whole band at $3.4 \mu \mathrm{m}$, the ratio of the integrated band intensity of the whole aliphatic band of the $3.4 \mu \mathrm{m}$ feature to that of the aromatic band at $3.3 \mu \mathrm{m}$ decreases sharply with UV irradiation as shown in Table 2. The reduction of the $3.4 \mu \mathrm{m}$ band results from the realization of hydrogen-atoms from the HAC material. This indicates the formation of more and larger aromatic structures. Quantitatively, this reduction $\left(R_{3.4}\right)$ as shown in Table 2 was estimated from the following equation,

$\operatorname{Re}_{3.4}=\frac{A_{\text {non }}-A_{\mathrm{UV}}}{A_{\text {non }}}$,

where the areas $A_{\text {non }}$ and $A_{\mathrm{UV}}$ are the whole integrated band intensities of the $3.4 \mu \mathrm{m}$ stretching band of the non-processed and UV-processed spectra, respectively. The values of $R e_{3.4}$ changes from $32 \%$ for S24 with lower UV irradiation to $76 \%$ for S26 with higher UV irradiation. Comparing of the variation of this band of the hydrogenated carbon grains (Mennella et al. 2001) and aliphatic hydrocarbon molecules (Muñoz-Caro et al. 2001), one sees a similar reduction of this band with the UV irradiation. Muñoz-Caro and collaborators have simulated analogs of carbonaceous dust grains in dense and diffuse interstellar medium. Mennella et al. (2001) have found that the irradiated hydrogenated carbon grains coated with $\mathrm{H}_{2} \mathrm{O}$ or $\mathrm{H}_{2} \mathrm{O}+\mathrm{CO}+\mathrm{NH}_{3}$ ice layer (as an analog of dust in the dense interstellar medium) have higher reduction than those coated with the Ar ice layer (as an analog of dust in the diffuse interstellar medium). On the other hand, for a similar analog for dust in the dense and diffuse interstellar medium, Muñoz-Caro et al. (2001) have found that hexane samples covered with $\mathrm{H}_{2} \mathrm{O}$ ice layer have lower reduction than those covered with the Ar ice layer. For hexane samples with a similar cap of $\mathrm{H}_{2} \mathrm{O}$ ice layer, the behavior of this reduction is reversely correlated with the carbon column density.

In the region from $5.5 \mu \mathrm{m}$ to $8.0 \mu \mathrm{m}$ as shown in Fig. 5, there are many strong features observed in the spectrum of S26 before and after the UV processing. One is the aromatic $\mathrm{C}=\mathrm{C}$ stretching vibration at about $6.2 \mu \mathrm{m}$, for which the fitting has given a band position at $6.27 \mu \mathrm{m}$. The relative strength of this band increases after the UV processing indicating that the materials become more graphitic by the UV irradiation for longer time. This result agrees with the first appearance of the $6.2 \mu \mathrm{m}$ band after UV irradiation of HAC films by Ogmen \& Duley (1988). Other bands are the aliphatic bending (deformation) $\mathrm{C}-\mathrm{H}$ vibrations near 6.85 and $7.25 \mu \mathrm{m}$. The strength of these deformation bands decreases after UV irradiation as shown in particular in the spectra of $\mathrm{S} 26$. In addition to the $\mathrm{C}=\mathrm{C}$ stretching and the $\mathrm{C}-\mathrm{H}$ bending vibrations, there are $\mathrm{C}=\mathrm{O}$ stretching vibration modes around $5.8 \mu \mathrm{m}$. These modes include sub-bands at 5.8, 5.96 and $6.15 \mu \mathrm{m}$ which become stronger after the UV processing. As we mentioned above, due to the contamination caused by oxygen atoms, the oxidation is probably very efficient and 
Table 2. The ratio of the intensity of aliphatic to aromatic bands.

\begin{tabular}{|c|c|c|c|c|c|c|c|c|}
\hline & \multicolumn{4}{|c|}{ S24 } & \multicolumn{4}{|c|}{ S26 } \\
\hline & $R e_{3.4}$ & $A_{3.4} / A_{3.3}$ & $A_{6.9} / A_{6.2}$ & $A_{7.27} / A_{6.2}$ & $R e_{3.4}$ & $A_{3.4} / A_{3.3}$ & $A_{6.9} / A_{6.2}$ & $A_{7.27} / A_{6.2}$ \\
\hline non-p ${ }^{(1)}$ & $\ldots$ & 13.5 & 2.7 & 0.46 & $\ldots$ & 11.9 & 4.35 & 0.56 \\
\hline UV-p ${ }^{(2)}$ & 0.31 & 10.6 & 0.45 & 0.04 & 0.76 & 5.0 & 0.34 & 0.08 \\
\hline
\end{tabular}

Notes. ${ }^{(1)}$ non-processed sample. ${ }^{(2)}$ the UV dose is $2.1 \times 10^{22} \mathrm{eV} \mathrm{cm}^{-2}$ for $\mathrm{S} 24$ and $1.0 \times 10^{23} \mathrm{eV} \mathrm{cm}^{-2}$ for S26.

can be triggered by the UV irradiation. As listed in Table 1, the band positions of some bands at 5.80, 6.27 and $7.79 \mu \mathrm{m}$ move to longer wavelengths $(5.84,6.30$ and $7.85 \mu \mathrm{m}$, respectively) after UV irradiation while others such as $6.15 \mu \mathrm{m}$ and $7.36 \mu \mathrm{m}$ move to shorter wavelengths $(6.12 \mu \mathrm{m}$ and $7.33 \mu \mathrm{m}$, respectively). The band positions at 5.96, 6.9 and $7.65 \mu \mathrm{m}$ mostly remain constant under the UV irradiation. The appearance of new small bands at $6.24,7.1$ and $7.46 \mu \mathrm{m}$ can be observed while the new small band at $5.7 \mu \mathrm{m}$ may appear due to the oxidation after the UV processing.

As shown in Table 2, the ratios of the strengths of aliphatic bands at $6.85 \mu \mathrm{m}$ and $7.25 \mu \mathrm{m}$ to the aromatic band at $6.2 \mu \mathrm{m}$ decrease after UV irradiation, referring to the transformation to aromatic structures in HAC materials. The calculations in this table were made considering the bands at $6.27 \mu \mathrm{m}$ and $6.3 \mu \mathrm{m}$ for the non-processed and UV-processed, respectively, of S26.

\subsection{The $10-17 \mu m$ spectral range}

In the fingerprint region from $10 \mu \mathrm{m}$ to $17 \mu \mathrm{m}$, many out-ofplane bands were observed in the spectra of non-processed and UV-processed HAC materials (S26). The Gaussian fitting parameters of these bands and their assignment are summarized in the bottom part of Table 1. In the spectrum of the non-processed HAC material as shown in Fig. 6 (top panel), the recognizable bands at $11.65 \mu \mathrm{m}$ (solo/duo) and $12.46 \mu \mathrm{m}$ (trio) are very strong. They are attributed to the $\mathrm{sp}^{2}$ aromatic $=\mathrm{C}-\mathrm{H}$ out-ofplane bending. Other bands at $15.87 \mu \mathrm{m}$ (strong) and at $16.63 \mu \mathrm{m}$ are attributed to $\mathrm{sp}^{2}$ aromatic $-\mathrm{C}-\mathrm{C}-\mathrm{C}$ in-plane bending modes (Van Kerckhoven et al. 2000). There are also small bands that are attributed to aromatic $=\mathrm{C}-\mathrm{H}$ out-of-plane bending such as those at $11.02 \mu \mathrm{m}$ (solo), $12.17 \mu \mathrm{m}$ and $12.9 \mu \mathrm{m}$ (duo and trio, respectively), and $13.23 \mu \mathrm{m}$ and $13.67 \mu \mathrm{m}$ (trio/quinto), while the band at $14.44 \mu \mathrm{m}$ (trio/quinto) is due to aromatic $-\mathrm{C}-\mathrm{C}-\mathrm{C}$ out-of-plane bending. Bands at $15.4 \mu \mathrm{m}$ and $16.3 \mu \mathrm{m}$ are probably due to the aromatic $-\mathrm{C}-\mathrm{C}-\mathrm{C}$ in-plane bending.

As shown in Fig. 6 (bottom panel), UV irradiation of HAC materials has a strong effect on both the strength and localization of bands in the fingerprint region. The peak positions of bands at $12.17,12.46,12.9,13.23,13.67$ and $15.4 \mu \mathrm{m}$ are moving toward shorter wavelengths to center at 12.14, 12.36. $12.64,13.04,13.41$ and $15.29 \mu \mathrm{m}$, respectively, while the peaks at 11.02 and $11.65 \mu \mathrm{m}$ show a redshift to 11.1 and $11.7 \mu \mathrm{m}$, respectively. The aromatic $=\mathrm{C}-\mathrm{H}$ out-of-plane bending bands at $11.35 \mu \mathrm{m}$ (solo) and $\mathrm{sp}^{2}$ aromatic $=\mathrm{C}-\mathrm{H}$ or $-\mathrm{C}-\mathrm{C}-\mathrm{C}$ out-ofplane bending at both $13.86 \mu \mathrm{m}$ and $14.29 \mu \mathrm{m}$ appear as new bands but bands at 14.44, 15.87 and 16.3, and $16.63 \mu \mathrm{m}$ have disappeared. The solo band at $11.35 \mu \mathrm{m}$ is related to the long straight edges in the PAHs while other modes are located at the corners (Tielens 2008).

The overall ratio of the solo band relative to others does not significantly change after the irradiation, although the band shifts seem to indicate an increasing size of aromatic units. This is no contradiction because large aromatic structures can have relatively few solo sites if they lack long zigzag edges (Hony et al. 2001).

\section{Astronomical implications}

Experimental studies of carbonaceous components in nano-sized structure have given astronomers a promising vision of the composition of the interstellar dust grains. This vision is very helpful to investigate the astronomical features shown in the observed spectra. Laboratory investigations of these features reveal the physical and chemical properties of the nano-solids, which are similar to those populating the interstellar space. In this section, some observational spectra are compared with those of the laboratory results of the HAC materials. These results show the role of the UV irradiation as an important mechanism for transformation of dust structures, which may be similarly taking place in the interstellar space.

Of course, not only the HAC materials can produce some of the astronomical features but there are also other carbonaceous materials such as PAHs and other materials like silicate-containing dust. The HAC materials are characterized by IR bands at about 3.04, $3.31 \mu \mathrm{m}$, the $\mathrm{C}-\mathrm{H}$ stretching band at $3.4 \mu \mathrm{m}$ and some bands at $6.27,6.9,7.27,7.9,11.66,12.41$, $13.1,13.9$ and $15.82 \mu \mathrm{m}$. These values are taken from the spectra of S26 as summarized in Table 1. In observed spectra of different lines of sight of the interstellar medium, $\mathrm{sp}^{3}$ aliphatic hydrocarbons can be detected at 3.4, 6.85 and $7.25 \mu \mathrm{m}$, whereas $\mathrm{sp}^{2}$ aromatic hydrocarbons can be observed at $3.28 \mu \mathrm{m}$ and $6.2 \mu \mathrm{m}$ of $=\mathrm{CH}$ and $\mathrm{C}=\mathrm{C}$ stretching modes, respectively, toward the galactic center (Chiar et al. 2000). For the $6.2 \mu \mathrm{m}$ band, the astronomical observations (Sloan et al. 2005, 2007) have shown that there are three band positions corresponding to A, B and C-class PAHs. In addition to the unidentified bands in these observations, several out-of-plane PAH-bands such as 11.3, 12.0, and $12.7 \mu \mathrm{m}$ have been observed.

\subsection{Interstellar aliphatic to aromatic transformation in relation with the UV radiation}

Experimental results have demonstrated the aliphatic to aromatic transformation caused by the UV irradiation of HAC materials by a change in relative band strengths as shown in Table 2. A similar transformation has been observed in features of astronomical objects (Pendleton \& Allamandola 2002). The IR emission features of hot-star sources embedded in a region near (within a projected distance of $2 \mathrm{pc}$ ) the $\mathrm{Sgr} \mathrm{A}^{*}$ object have been extensively used to characterize dust grain materials in the diffuse ISM (Sandford et al. 1991). The Galactic center region ( $3 \mathrm{pc}$ in diameter) is surrounded by a higher density ring of gas and dust (Güsten et al. 1987), thus the extinction is generally caused by the materials in the diffuse and dense ISM. Stretching vibrations at $3.4 \mu \mathrm{m}$ and the bending vibrations at $6.85 \mu \mathrm{m}$ 


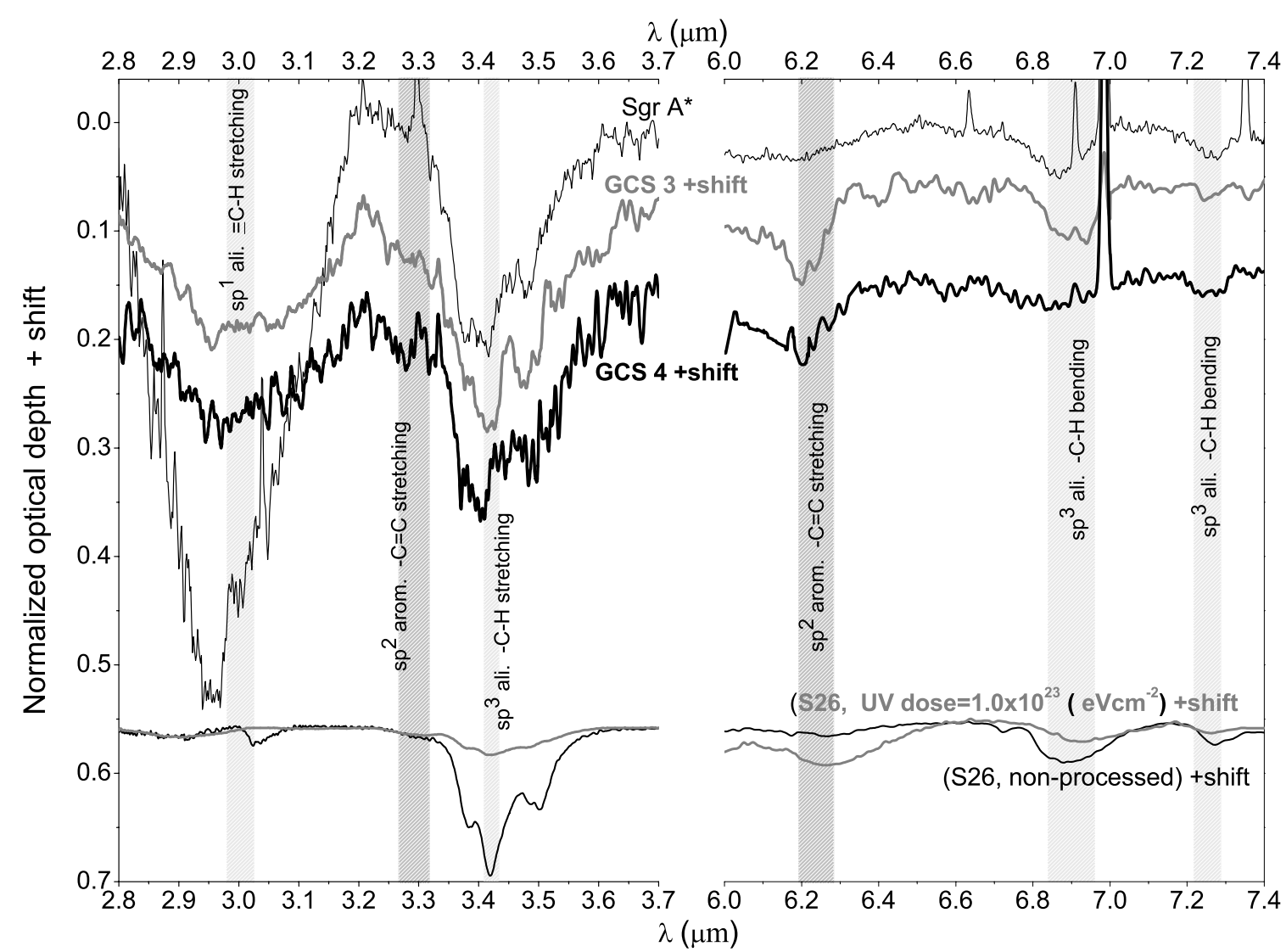

Fig. 7. Aliphatic and aromatic infrared absorption bands in spectra (ISO-SWS (AOT1)) toward the galactic center (Sgr A*, GCS 3 and GCS 4, Chiar et al. 2000) compared to those of the laboratory samples (S26) before and after UV irradiation.

and $7.25 \mu \mathrm{m}$ are assigned to a population of very small unaligned HAC grains in diffuse medium. These absorption features in addition to those of aromatic hydrocarbons at $3.28 \mu \mathrm{m}$ and $6.2 \mu \mathrm{m}$ (=C-H and $-\mathrm{C}=\mathrm{C}$ stretching modes, respectively) have also been observed along different lines of sight ( $\mathrm{Sgr} \mathrm{A*}$, GCS 3 and GCS 4) toward the Galactic center, see Chiar et al. (2000). These authors found that the optical depth along the sight line of Sgr A* is higher than that toward the sight lines of GCS 3 and GCS 4. Consequently, the Sgr A* spectrum characterizes the molecular cloud dust, whereas the spectra of GCS 3 and GCS 4 characterize the dust in sight lines through the diffuse medium. As summarized in Table 2 in Chiar et al. (2000), aliphatic bands toward Sgr $\mathrm{A}^{*}$ at 3, 3.4, 6.85, and $7.25 \mu \mathrm{m}$ have higher values of the optical depth $(0.50 \pm 0.01,0.21 \pm 0.01$, $0.05 \pm 0.01$ and $0.03 \pm 0.01$, respectively) than those of other objects. On the other hand, the optical depth of aromatic bands at $3.28 \mu \mathrm{m}$ and $6.2 \mu \mathrm{m}$ toward Sgr $\mathrm{A}^{*}$ has lower values $(<0.02$ and $0.05 \pm 0.01$, respectively) than those in the other sight lines. These variations in the strength of aliphatic bands to those in the aromatic bands are consistent with the variations in the laboratory results summarized in Table 2 after UV irradiation. We concluded that the aliphatic to aromatic transformation occurs because of the effect of the UV radiation. For the diffuse medium (represented by dust toward GCS 3 and GCS) the UV irradiation time-scale for the photodissociation of $\mathrm{C}-\mathrm{H}$ bonds is $1.2 \times$ $10^{6} \mathrm{yr}$ (Sorrell 1990). This is short compared to the lifetime of the diffuse cloud $\left(3 \times 10^{7} \mathrm{yr}\right)$ that grains spend (Jenniskens 1993). On the other hand, for the molecular medium (represented by dust toward Sgr $\mathrm{A}^{*}$ ) the UV irradiation time-scale, which is about $10^{9} \mathrm{yr}$, exceeds the cloud lifetime, which is about $2 \times 10^{8}$ yr (Greenberg 1986). Consequently, dehydrogenation of HAC materials would mainly take place in diffuse ISM with high UV radiation processing.

\subsection{The interstellar $3.4 \mu \mathrm{m}$ stretching}

At the high UV-irradiation doses of S26, the strength of the $3.4 \mu \mathrm{m}$ band decreases very much as shown in Fig. 7. In spite of the high aromaticity expected in the diffuse ISM due to the UV irradiation of HACs, the aliphatic bands $(3.4,6.85$ and $7.25 \mu \mathrm{m}$ ) are commonly observed. This discrepancy has been explained by Mennella et al. (2002). Under diffuse-medium conditions, they found that the time interval necessary to balance between the destruction rate by UV photons and the formation (hydrogenation) rate by $\mathrm{H}$ atoms of $\mathrm{C}-\mathrm{H}$ bonds is $10^{4} \mathrm{yr}$. This interval is approximately three orders of magnitude shorter than the typical time scale of the diffuse ISM $\left(3 \times 10^{7} \mathrm{yr}\right)$. These authors found that the ratio of the destruction rate to the formation rate of aliphatic $\mathrm{C}-\mathrm{H}$ bonds is 0.53 . In the reflection nebulae NGC 1333 svs 3 and NGC 2023, Joblin et al. (1996) found that the intensity of the $3.29 \mu \mathrm{m}$ band is stronger than that of the $3.4 \mu \mathrm{m}$ band. They concluded that the UV radiation field is destroying weaker aliphatic bonds, which varyies the proportions of the aliphatic and aromatic units in their mixtures in interstellar hydrocarbons.

\subsection{The $6.2 \mu \mathrm{m}$ band of $H A C$ in relation with the $B$ and $\mathrm{C}$-class $\mathrm{PAHs}$}

Indeed, the theoretical model developed by Jones et al. (1990) has suggested that HAC materials contain PAH clusters 


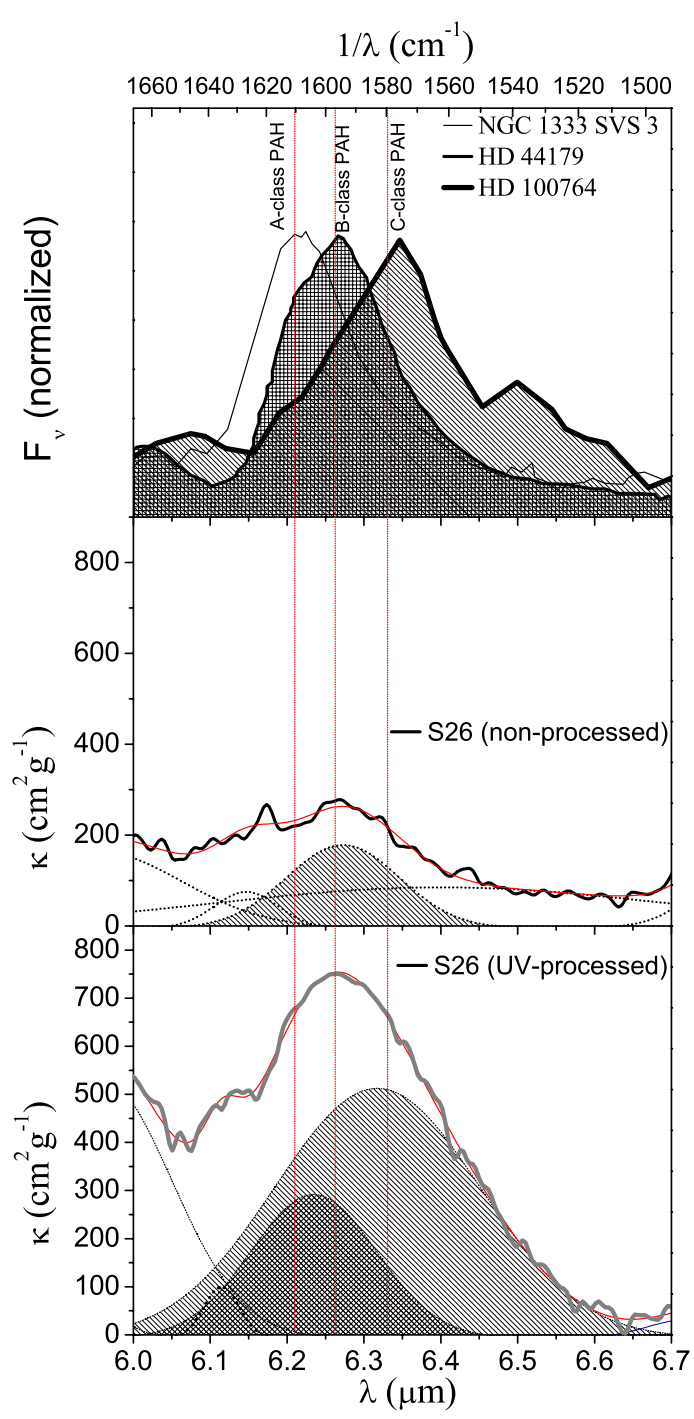

Fig. 8. $6.2 \mu \mathrm{m}$ band of astronomical features (top panel) of the NGC 1333 svs 3 (A-class PAH), HD 44179 (B-class PAH), and DH 100764 (C-class PAH), and that of the experimental spectra of both non-processed (middle panel) and UV-processed (bottom panel) HACs. The hatched regions in the top panel are peaks of the B- and C-classes while in the middle and the bottom they are the corresponding peaks from the laboratory data. The vertical lines represent the band positions of the $\mathrm{C}=\mathrm{C}$ structure at $6.21,6.27$ and $6.3 \mu \mathrm{m}$ of the interstellar spectra of A-, B- and C-classes PAHs, respectively.

embedded within a matrix of aliphatic-bonded hydrocarbons. Experimentally, Scott et al. (1997) showed that HAC materials may be the source of large PAH and fullerene molecules. These suggestions agree with the point of view of the study performed by Sloan et al. (2007). Yhe HAC materials reveal C-class PAH when they are exposed to a less intense UV radiation field than that of the materials revealing the A- and B-class PAHs. In this study, the NGC 1333 svs 3 (A-class PAH), HD 44179 (B-class PAH), and DH 100764 (C-class PAH) have effective temperatures of $14000 \mathrm{~K}, 9520 \mathrm{~K}$ (Kenyon \& Hartmann 1995), and $4850 \mathrm{~K}$ (Dominy 1984) and have spectral types of B6, A0 III and $\mathrm{C} 1,1$, respectively.

In the comparison with the observed spectra of the above astronomical objects as shown in the top panel of Fig. 8, we focused on the B- and C-class PAHs that could match our experimental results, but the A-class PAH does not match ours. The experimental result in the middle panel for the nonprocessed sample shows the $6.27 \pm 0.02 \mu \mathrm{m}$ band (weak), which is very broad relative to its small strength. This band is approximately in a range between $\mathrm{B}$ - and C-lass PAHs, and if we take the fitting errors into account, it falls in the B-class range. It probably originates from the aromatic $\mathrm{C}=\mathrm{C}$ structures formed in the hot plasma during the production process of the $\mathrm{HAC}$ materials by the laser ablation.

After UV irradiation as shown in the bottom panel, we have two positions of the $\mathrm{C}=\mathrm{C}$ stretching at $6.24 \pm 0.01$ and $6.3 \pm$ $0.07 \mu \mathrm{m}$. The first one at $6.24 \pm 0.01 \mu \mathrm{m}$ (consistent with B-class) is expected to originate from the UV irradiation of the lesser amount of aromatic structures, which was formed in the non-processed sample. The second position at $6.3 \pm 0.07 \mu \mathrm{m}$ (consistent with C-class) is a new band caused by the UV irradiation of aliphatic structures. Its width looks broader than that of the observed band. This broadening is attributed to the role of aliphatic modes, which are still mixed after the UV irradiation and contribute to the absorption in the irradiated spectrum. The presence of these aliphatic modes is confirmed from their spectroscopic signatures after UV irradiation as listed in Table 1.

Sloan et al. (2007) have suggested that the $6.3 \mu \mathrm{m}$ (C-class) originates from the mixtures of aromatic and aliphatic materials that have not yet been subjected to strong UV radiation. These authors had seen that the aliphatic bonds are first broken once they are exposed to harsh UV irradiation. Consequently, the more fragile aliphatic bonds may survive. Basically, the aliphatic or fragile aliphatic bonds may cause the band to be still broader. According to Sloan et al.'s suggestion, in which the materials reveal that the A- and B-class PAHs are exposed to higher UV radiation, we have no possibilities in our case to irradiate to higher values of UV doses or to realize the uniformity in irradiation to observe a shift toward the A- and B-classes.

In conclusion, if the irradiated HACs are still containing both the aromatic and aliphatic structures (with different fractions), we have one broad band of the two bands of B- and C-classes. Then experimentally the effect of UV irradiation on the $6.2 \mathrm{mi}-$ cron is absolutely clear in its intensity while observing a shift in the band position may require uniform and intense UV irradiation. The small peaks at $6.15 \mu \mathrm{m}$ and $6.12 \mu \mathrm{m}$ in the experimental spectra in the middle and bottom panels, respectively, of Fig. 8 could be due to the contaminations of oxygen-bearing molecules $\left(\mathrm{H}_{2} \mathrm{O}\right.$ and $\left.\mathrm{CO}\right)$.

\subsection{The out-of-plane bands}

Relative to the standard out-of-plane bands of PAHs in the diffuse ISM at 11.3 (solo), 12.0 (duo) and 12.7 (trio) $\mu \mathrm{m}$, observations (Sloan et al. 2007) toward objects showing the C-class PAH features have found a remarkable redshift of the band positions. As shown in the top panel of Fig. 9, these bands, as seen in HD 100764, agree with some shaded experimental bands of the non-processed HAC material. In the bottom panel, the out-of-plane band positions of NGC 1333 svs 3 (A-class PAH) and HD 44179 (B-class PAH) (Sloan et al. 2007) come very close to the standard positions at $11.3,12.0,12.7$, and $13.7 \mu \mathrm{m}$. These bands agree with those of some shaded bands in the UV-processed spectrum. The appearance of the $11.35 \mu \mathrm{m}$ feature in the spectrum of the laboratory-irradiated material indicates the stronger aromaticity in HAC materials after the UV irradiation. It seems that the behavior of this band is similar to that of the $6.2 \mu \mathrm{m}$ band with UV irradiation. Its appearance is related to the plenty of the $\mathrm{C}=\mathrm{C}$ structure, which might be similar to that in the A- and B-classes PAHs. 


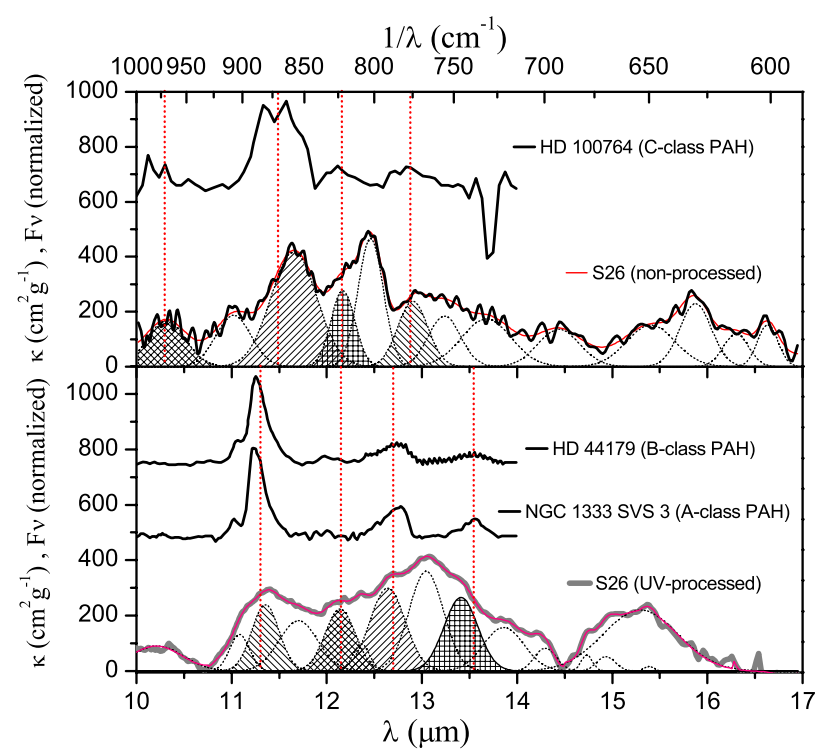

Fig. 9. Out-of-plane bands in the range of $10 \mu \mathrm{m}$ to $17 \mu \mathrm{m}$ compared to those of the interstellar PAHs. The vertical lines represent the band positions of out-of-plane bands of the interstellar spectra.

In our study we found a higher ratio of duo/trio/quarto to solo bands compared to the observed out-of-plane bands, both before and after irradiation. This ratio does not reflect the size and abundance of aromatic units but their shape and symmetry. The preference of the $11.3 \mu \mathrm{m}$ band in observed spectra requires ribbon-like structures (Hony et al. 2001), which are clearly not dominating in our samples. Nevertheless, the band shifts and the appearance of the $11.35 \mu \mathrm{m}$ feature indicate that larger PAH clusters can be formed once UV radiation breaks up the $\mathrm{C}-\mathrm{H}$ bonds in HAC materials. The increase of the graphene layer lengths measured by HRTEM (Gadallah et al. 2011) supports our interpretation of the IR spectral changes as evidence of an increase in aromaticity.

The UV radiation field in the ISM is thus expected to increase the aromaticity of the hydrocarbon structure. This is in principle consistent with the trend between the A and B-class PAHs and the C-class PAH (Sloan et al. 2007), for which the $11.3 \mu \mathrm{m}$ band position has redshifted as the effective temperature decreases. Bands at $13.41 \mu \mathrm{m}$ and $14.29 \mu \mathrm{m}$ (Table 1) have also been observed after UV irradiation, which are close to the $13.5 \mu \mathrm{m}$ and $14.2 \mu \mathrm{m}$ bands observed toward many astronomical objects, as shown in Fig. 3 in Hony et al. (2001).

\section{Conclusions}

The mid-IR analysis of experimental spectra of non-processed and UV-processed HAC materials can provide much information about the carriers of observed spectroscopic features in the diffuse ISM. The variability in strength of aliphatic (stretching and deformation) bands relative to that of the aromatic bands reveals the aliphatic to aromatic transformation of nano-sized hydrocarbons by UV irradiation, which is about $33 \%$ of the average of the interstellar $\mathrm{UV}$ dose $\left(3 \times 10^{23} \mathrm{eV} \mathrm{cm}^{-2}\right)$ during the lifetime of the diffuse cloud $\left(3 \times 10^{7} \mathrm{yr}\right)$ (Jenniskens 1993). This kind of UV processing can explain the evolution of the structure of HACs in the ISM, which could be the source of the PAH clusters. The standard out-of-plane bands observed due to the presence of PAHs in the diffuse ISM can be recognized after the UV-processing.

Acknowledgements. We are grateful to the laboratory group at Astrophysical Institute and University Observatory, Friedrich Schiller University Jena, Germany. We are also grateful to the referee V. Mennella for his valuable comments and suggestions.

\section{References}

Allamandola, L. J., Tielens, A. G. G. M., \& Barker, J. R. 1989, ApJS, 71, 733 Angus, J. C., \& Jansen, F. 1988, J. Vacuum Sci. Technol., 6, 1778

Chiar, J. E., Tielens, A. G. G. M., Whittet, D. C. B., et al. 2000, ApJ, 537, 749 Chiar, J. E., Peeters, E., \& Tielens, A. G. G. M. 2002, ApJ, 579, L91 Dartois, E., \& Muñoz-Caro, G. M. 2007, A\&A, 476, 1235

Dischler, B., Bubenzer, A., \& Koidl, P. 1983, Appl. Phys. Lett., 42, 636 Dominy, J. F. 1984, ApJS, 55, 27

Draine, B. T., \& Li, A. 2001, ApJ, 551, 807

Gadallah, K. A. K., Mutschke, H., \& Jäger, C. 2011, A\&A, 528, A56

Greenberg, J. M. 1986, Ap\&SS, 128, 17

Grishko, V. I., \& Duley, W. W. 2002, ApJ, 568, 448

Güsten, R., Genzel, R., Wright, M. C. H., et al. 1987, ApJ, 318, 124

Hony, S., Van Kerckhoven, C., Peeters, E., et al. 2001, A\&A, 370, 1030

Hu, A., \& Duley, W. W. 2007, ApJ, 660, L137

Hudgins, D. M., \& Allamandola, L. J. 1999, ApJ, 516, L41

lida, Y., \& Yeung, E. S. 1994, Appl. Spectrosc., 48, 945

Jäger, C., Mutschke, H., Henning, T., \& Huisken, F. 2008, ApJ, 689, 249

Jenniskens, P. 1993, A\&A, 274, 653

Joblin, C., Tielens, A. G. G. M., Allamandola, L. J., \& Geballe, T. R. 1996, ApJ, 458,610

Jones, A. P., Duley, W. W., \& Williams, D. A. 1990, QJRAS, 31, 567

Kenyon, S. J., \& Hartmann, L. 1995, ApJS, 101, 117

Kwok, S., Volk, K., \& Hrivnak, B. J. 1999, A\&A, 350, L35

Mckenzie, D. R., Mcphedran, R. C., Savvides, N., \& Botten, L. C. 1983, Philosophical Magazine Part B, 48, 341

Mennella, V., Muñoz Caro, G. M., Ruiterkamp, R., et al. 2001, A\&A, 367, 355

Mennella, V., Brucato, J. R., Colangeli, L., \& Palumbo, P. 2002, ApJ, 569, 531

Muñoz-Caro, G. M., Ruiterkamp, R., Schutte, W. A., Greenberg, J. M., \& Mennella, V. 2001, A\&A, 367, 347

Ogmen, M., \& Duley, W. W. 1988, ApJ, 334, L117

Papoular, R., Conrad, J., Giuliano, M., Kister, J., \& Mille, G. 1989, A\&A, 217, 204

Peeters, E. 2011, in EAS Publ. Ser. 46, eds. C. Joblin, \& A. G. G. M. Tielens, 13

Peeters, E., Hony, S., Van Kerckhoven, C., et al. 2002, A\&A, 390, 1089

Pendleton, Y. J., \& Allamandola, L. J. 2002, ApJS, 138, 75

Pendleton, Y. J., Sandford, S. A., Allamandola, L. J., Tielens, A. G. G. M., \& Sellgren, K. 1994, ApJ, 437, 683

Puget, J. L., \& Leger, A. 1989, ARA\&A, 27, 161

Sakata, A., Wada, S., Onaka, T., \& Tokunaga, A. T. 1987, ApJ, 320, L63

Sandford, S. A., Allamandola, L. J., Tielens, A. G. G. M., et al. 1991, ApJ, 371, 607

Scott, A., Duley, W. W., \& Pinho, G. P. 1997, ApJ, 489, L193

Sloan, G. C., Keller, L. D., Forrest, W. J., et al. 2005, ApJ, 632, 956

Sloan, G. C., Jura, M., Duley, W. W., et al. 2007, ApJ, 664, 1144

Sorrell, W. H. 1990, MNRAS, 243, 570

Tielens, A. G. G. M. 2008, ARA\&A, 46, 289

Van Kerckhoven, C., Hony, S., Peeters, E., et al. 2000, A\&A, 357, 1013

Whittet, D. C. B., Boogert, A. C. A., Gerakines, P. A., et al. 1997, ApJ, 490, 729 